\title{
Capsule Commentary on Goddu et. al., Do Words Matter? Stigmatizing Language and the Transmission of Bias in the Medical Record
}

\author{
Jason Schnittker, PhD \\ University of Pennsylvania, Philadelphia, PA, USA. \\ $\mathrm{J}$ Gen Intern Med 33(5):748 \\ DOI: $10.1007 / \mathrm{s} 11606-018-4357-2$ \\ (c) Society of General Internal Medicine 2018
}

G oddu et al. ${ }^{1}$ provide compelling evidence that the words used to describe patients in medical records matter. Their research design is imprecise, however, and the study is better at demonstrating the importance of words generally than in identifying what words matter or why.

The study employs two arms: one employing neutral language and the other employing stigmatizing language. The two arms differ in many ways, however, including but hardly limited to stigma. For instance, the stigma arm also introduces information that could be clinically relevant. Surely, as the authors argue, describing someone as using narcotics is different from describing them as using opioid pain medication. Yet the stigma arm goes much further: it adds that Mr. R is "narcotic dependent" and leaves ambiguous, in a way that is not true of the neutral language chart, whether he visits the ED for the receipt of pain medication or for something else. In addition, the stigma arm describes different kinds of pain, not just a different description of the same pain. The stigma arm reports that Mr. R has 10/10 pain, whereas the neutral arm only reports episodic pain, of 8 to 10 crises per year. Other decisions by the investigators contaminate the design in different ways. The study introduces information on socioeconomic status, which the authors stipulate is "irrelevant." That may be true, but the study leaves unclear whether the source of the

Published online February 15, 2018 stigma was lower socioeconomic status or how that lower socioeconomic status was described. In the stigma arm, the patient is revealed to be using the services of the housing authority, something far beyond ordinary lower socioeconomic status.

A key insight of modern social psychology is that small changes can make a big difference in person perception. Reading this study, clinicians might be tempted to infer that so long as they avoid overt and frequent stigmatizing language they will prevent the transmission of bias. But the problem is deeper: it is possible that describing one patient as narcotic dependent was alone sufficient to foster negative attitudes, especially given the persistent stigma surrounding psychiatric disorders. $^{2}$

Corresponding Author: Jason Schnittker, PhD; University of Pennsylvania, Philadelphia, PA, USA (e-mail: jschnitt@ssc.upenn.edu).

Compliance with ethical standards:

Conflict of interest: The author has no conflicts of interest with this article.

\section{REFERENCES}

1. Goddu A, O’Conor KJ, Lanzkron S, Saheed MO, Saha S, Peek ME, Haywood C, Beach MC. Do words matter? Stigmatizing language and the transmission of bias in the medical record. $J$ Gen Intern Med. DOI: https://doi.org/10.1007/s11606-017-4289-2

2. Schnittker J. An uncertain revolution: why the rise of a genetic model of mental illness has not increased tolerance. Soc Sci Med 67, 1370-1381. 